\title{
Non-contact PIM Measurement Method for Antenna using High- level IM Standard
}

This paper was downloaded from TechRxiv (https://www.techrxiv.org).

\section{LICENSE}

CC BY-SA 4.0

SUBMISSION DATE / POSTED DATE

16-11-2021 / 19-11-2021

\section{CITATION}

Kuwata, Masayoshi; kuga, Nobuhiro (2021): Non-contact PIM Measurement Method for Antenna using Highlevel IM Standard. TechRxiv. Preprint. https://doi.org/10.36227/techrxiv.17020016.v1

DOI

10.36227/techrxiv.17020016.v1 


\title{
Non-contact PIM Measurement Method for Antenna using High-level IM Standard
}

\author{
Masayoshi Kuwata, Student Member, IEEE, and Nobuhiro Kuga, Member, IEEE
}

\begin{abstract}
A non-contact passive intermodulation distortion (PIM) measurement method for antenna using high-level IM standard (HIMS) is described as proposed herein. A novel method to estimate coupling loss between sensing antenna and a sample antenna is proposed, after which a complete one-port non-contact measurement for antenna is realized. Measurements are conducted in three stages: measuring IM characteristics of HIMS, estimating coupling loss using HIMS, and estimating practical PIM level of a sample antenna. A high-level IM standard consisting of a Schottky barrier diode was used for this study. The method enables ready evaluation of practical PIM of a sample antenna from a complete one-port non-contact measurement with excellent stability and repeatability. Then the validity of the proposed method was confirmed experimentally using a printed dipole antenna. After presenting an applicable measurement method for array antenna incorporating the proposed method, its effectiveness was verified experimentally.
\end{abstract}

Index Terms-antenna measurement, non-contact measurement, nonlinear noise, passive intermodulation

\section{INTRODUCTION}

Intermodulation distortion (IM) is a spurious wave produced when multiple frequencies are applied to a nonlinear device, in which one caused by residual nonlinearity in passive linear devices is designated as passive intermodulation distortion (PIM). Actually, PIM is much weaker than IMs of nonlinear devices. However, PIM often presents severe difficulties in base station antennas of mobile communication systems because it is not negligible in comparison with weak uplink signals [1]-[5]. Investigations of their mechanisms have also been conducted for PIM sources such as printed circuits and for coaxial connectors and waveguide flanges [6]-[11].

Standard documents related to PIM measurements have been published by IEC [12]. As described in them, observed PIM is classified into two types: one is PIM propagating toward the transmitter, which is designated as reverse PIM (R-PIM); the other is propagating to a matched load, which is designated as forward PIM (F-PIM). Now related to a basestation antenna system, R-PIM is fundamentally important because it has possible interference with a weak uplink signal. Therefore, most earlier studies have used methods by which the antenna under test (AUT) is connected directly to a PIM tester for measurement of R-PIM or of R-IM [13]-[16]. As described herein, we designate the method as direct measurement (DM). Fig. 1(a) portrays a diagram of the DM environment.

The authors are with the Graduate School of Engineering Science, Yokohama National University, 79-5 Tokiwadai, Hodogaya-ku, Yokohama-shi, 2408501, Japan, e-mail: (kuwata-masayoshi-yv@ynu.jp).

J. Doe and J. Doe are with Anonymous University.

Manuscript received April 19, 2005; revised August 26, 2015.
This basic and simple method can evaluate PIMs of practical antennas that are shared in transmission and reception systems.

In fact, PIM measurement is sensitive to the contact state at metallic junctions, thus several non-contact PIM measurement methods have been proposed [17]-[19]. The methods reported in the literature [17], [18] were applied to large components such as antennas and microstrip lines. However, non-contact measurement was introduced partly for the PIM-detecting probe. A complete one-port non-contact measurement using a coaxial tube was proposed in earlier reports [19]. It had shortcomings in terms of the sample-size limitation because it used a coaxial tube. Therefore, it has been extended to larger samples such as antennas [20], [21]. As described herein, complete non-contact measurements for antennas are denoted as non-contact measurements (NCM). Actually, Fig. 1(b) is a diagram portraying the NCM concept. Using this method, AUT is excited by a sensing antenna connected to a PIM tester. Then the PIM signal re-radiated from the AUT is received by the PIM tester via the sensing antenna.

When using NCM for antenna PIM measurements, two major differences from DM must be considered. One is the component to be observed and evaluated. The other is the existence of the coupling loss between sensing antenna and AUT. The former is that the PIM signal observed physically in NCM is F-PIM radiated into space from AUT, which is not R-PIM in DM. However, DM can be estimated using NCM because the PIM power produced by a PIM source is split equally to the generator side and the termination side when the circuit is under a good matching condition [13]. For the latter, how the loss in practical measurement procedures is measured is important. The direct method is to use vector network analyzers (VNA) [21]. It should be done in practical power, which is much greater than that in popular microwave measurements. It is necessary for high precision measurements. However, that is an expensive process that is not cost-effective.

Based on the background presented above, this paper presents a proposal of a novel method to estimate the coupling loss using a high-level IM standard (HIMS) for non-contact PIM measurements for an antenna. It is beneficial that the proposed method does not need any expensive VNA measurement in practical power to obtain the coupling loss between AUT and sensing antenna. Its validity was confirmed based on this study through PIM measurement of a printed dipole antenna and its two-element array using a HIMS consisting of a diode. It is also valuable to undertake an examination of PIM in array antennas because few reports have described non-contact PIM measurement for array antennas, except for fundamental work 
on PIM localization [22].

The paper is organized as follows. In section 2, the concept of the proposed method is described through a PIM measurement of a printed dipole antenna. Results demonstrating its validity are presented. In section 3, the proposed method is shown through experimentation to be applicable to array antennas using a two-element array of the printed dipole antenna. Finally, this paper is concluded in section 4 .

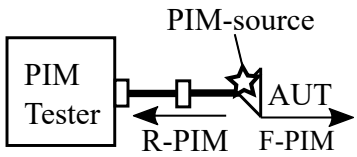

(a) DM: Direct Measurement

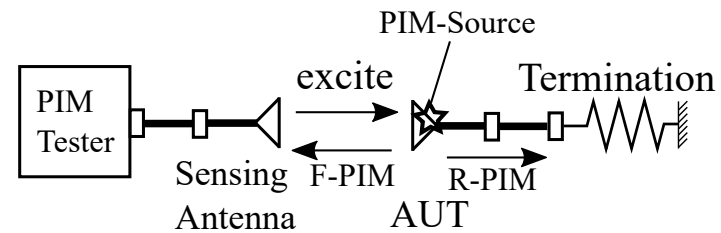

(b) NCM: Non-contact Measurement

Fig. 1. Diagrams of PIM measurements of two types for the antenna

\section{CONCEPT OF NCM USING HIMS}

As described in this paper, all experiments were conducted in the $2 \mathrm{GHz}$ band. The transmitting frequencies $f_{1}$ and $f_{2}$ are, respectively, $2.05 \mathrm{GHz}$ and $2.20 \mathrm{GHz}$. The observed PIM or IM is the third-order one at $f_{i m}=2 f_{1}-f_{2}=1.90 \mathrm{GHz}$.

Fig. 2 shows block diagrams of the proposed measurement systems. The measurement is conducted in three stages as shown in (a), (b), and (c), which are labeled respectively as the first, second, and third measurements. In the second and third measurements, AUT is coupled to a sensing antenna electromagnetically without direct connection, which implies non-contact measurement.

Fig. 3(a) presents the HIMS configuration used for this study. Because it is necessary to produce a dominant IM signal for the second measurement, it is desirable to have high nonlinear characteristics. A diode mounted in the center of a microstrip line (MSL) is used as HIMS. The dielectric substrate is $1.6 \mathrm{~mm}$ thick with relative dielectric constant $\epsilon_{r}=2.6$. The line width is adjusted to match $50 \Omega$. The diode used as HIMS for this study is the Schottky barrier diode (RB721Q-40; Rohm Co. Ltd.).

Fig. 3(b) depicts the configuration of PIM-source model used for this study. This model is used in an antenna or feeding circuit. It is tested later in the third measurement as the PIM source in AUT. Because it is necessary to produce a weaker IM signal than that of HIMS, a pair of parallel diodes is mounted in the middle of the MSL. The mounted diode, dielectric substrate, and all parameters are identical to those of HIMS.

Fig. 4 shows transmission and reflection characteristics of HIMS and the PIM-source model. At test frequencies $\left(f_{1}, f_{2}, f_{i m}\right)$, it was confirmed that both models have lowloss characteristics. In addition, Table I shows the observed
IM level when \#a is connected to the PIM tester and when \#b is terminated at applying $-10 \mathrm{dBm} /$ tone from the PIM tester. The observed IM level of the PIM-source model is weaker than that of HIMS by $18 \mathrm{~dB}$. Based on that finding, it can be confirmed that the HIMS and PIM-source model are a low-loss transmission line with high-level IM source, low-level PIM source, respectively, at test frequencies of $\left(f_{1}, f_{2}, f_{i m}\right)$. As described herein, the IM signal generated by the PIM-source model is denoted as PIM, whereas the one generated by HIMS is denoted as IM. Each will be described separately.

In the following, we explain details of each measurement stage with experimentally obtained results obtained using a basic single-element model.

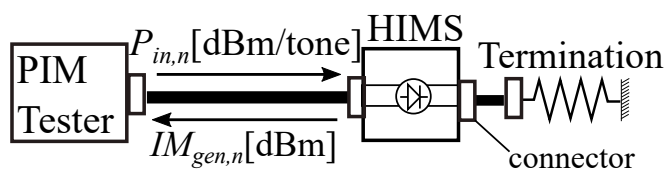

(a) First measurement system

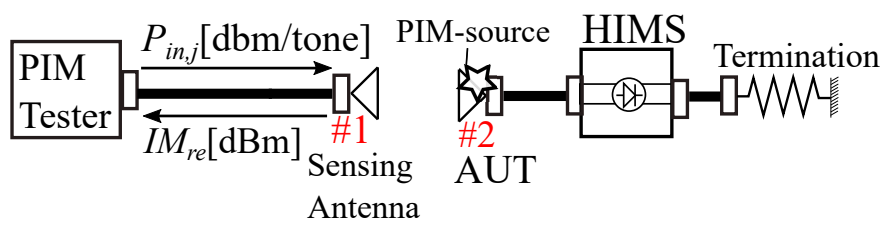

(b) Second measurement system

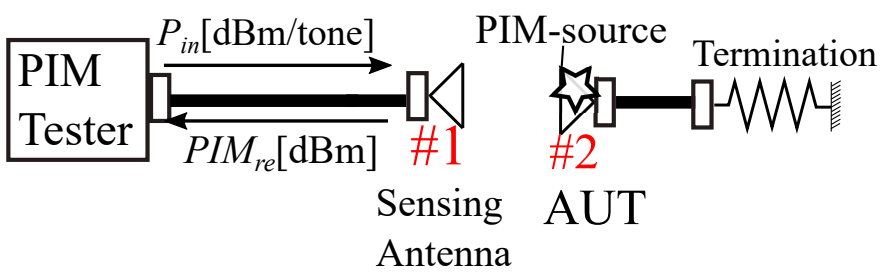

(c) Third measurement system

Fig. 2. Block diagrams of measurement systems of the proposed method.

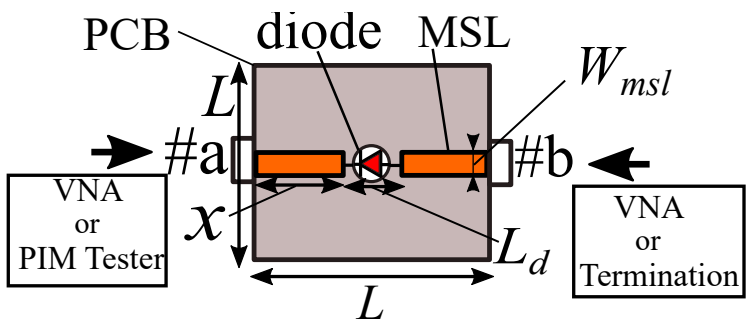

$W_{m s l}=4.4, L=50, L_{d}=4, x=23$ unit:mm

(a) HIMS

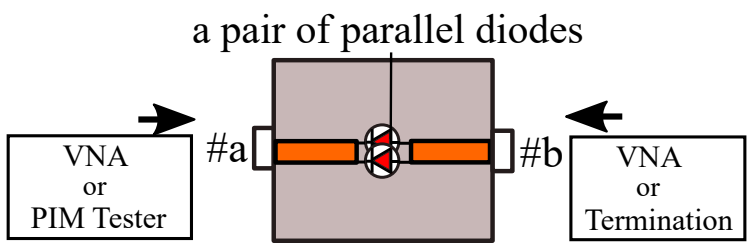

(b) PIM-source model

Fig. 3. Configurations of the HIMS and PIM-source model. 


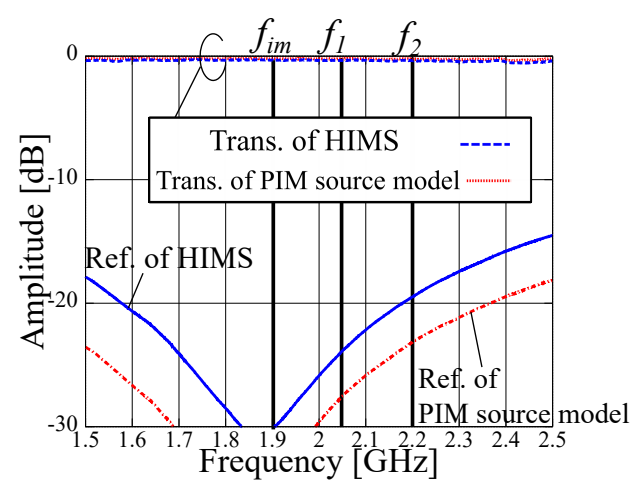

Fig. 4. Transmission (Trans.) and reflection (Ref.) characteristics of HIMS and PIM source model.

TABLE I

COMPARISON OF OBSERVED PIM LEVEL OF THE PIM-SOURCE MODEL WITH HIMS AT APPLYING 10 DBM/TONE

\begin{tabular}{c|c}
\hline Model & PIM level $[\mathrm{dBm}]$ \\
\hline \hline HIMS & -85.3 \\
PIM-source model & -104 \\
\hline
\end{tabular}

\section{A. Basic single-element model}

Fig. 5 portrays a configuration of sensing antenna \#1 and AUT \#2 when a single element printed dipole antenna is used as AUT. The patch antenna on the left side, which is used as sensing antenna, is identical to that used in studies reported in the literature [21]. The printed dipole antenna is also identical to that in the literature [21], [23] except for feeding-line structure, which is fabricated by the same dielectric substrate of HIMS. The MSL on the back side of the substrate couples electromagnetically to the antenna via the notch. The PIMsource model shown in Fig. 3(b) is used on the back side MSL for testing in the third measurement.

Fig. 6 presents reflection characteristics of the sensing antenna and AUT. At test frequencies $\left(f_{1}, f_{2}, f_{i m}\right)$, reflection coefficients are under $-10 \mathrm{~dB}$. This finding indicates that antennas are in a good matching condition.

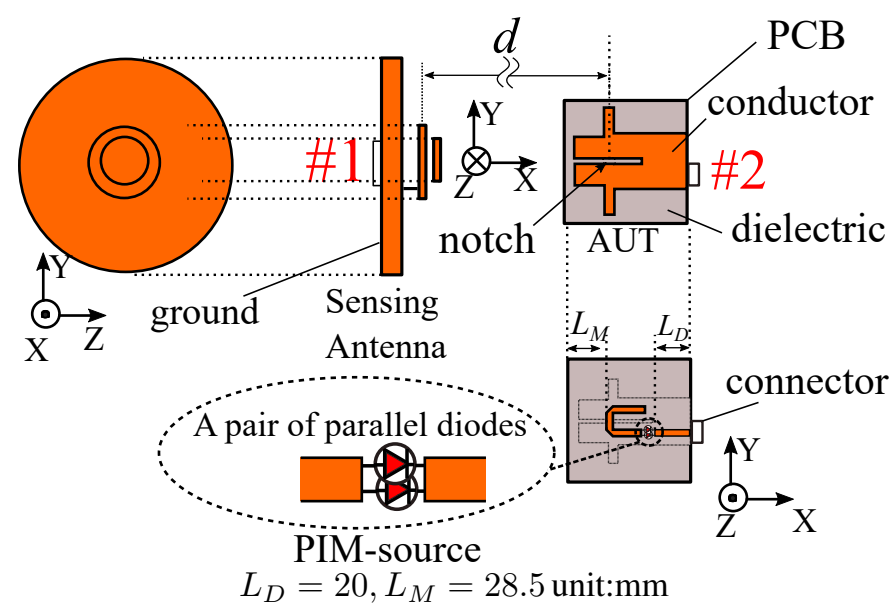

Fig. 5. Configurations of the sensing antenna and AUT with PIM-source model.

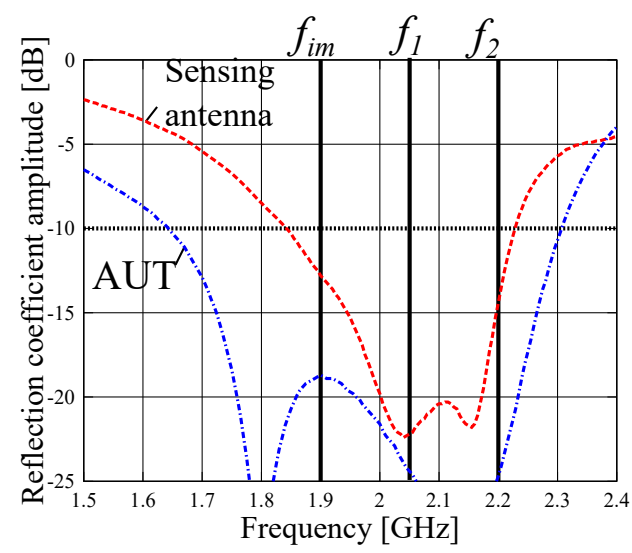

Fig. 6. Reflection coefficient of sensing antenna and AUT.

\section{B. First measurement}

In the first measurement shown in Fig. 2(a), HIMS is connected directly to the PIM tester to measure its IM characteristics. Specifically, the IM level generated by HIMS $I M_{g e n, n}[\mathrm{dBm}]$ is measured as a function of applied power $P_{i n, n}[\mathrm{dBm} / \mathrm{tone}]$, where "n" is an integer label denoting the measurement point $(n=1,2, \cdots)$. Then, an increase coefficient $C_{n}[\mathrm{~dB} / \mathrm{dB}]$, which is denoted as "power slope" in this paper, is given by the next equation.

$$
C_{n}=\frac{I M_{g e n, n+1}-I M_{g e n, n}}{P_{i n, n+1}-P_{i n, n}}
$$

Fig. 7 portrays a measured IM level $I M_{g e n, n}$ (blue line). The power slope $C_{n}$ (red line) is calculated based on equation (1) using the measured IM level $I M_{\text {gen, } n}$. It shows that the power slope $C_{n}$ fluctuates over $P_{i n, n}=-2 \mathrm{dBm} /$ tone, although it remains at approximately 3 in a small input-power range. For this reason, power slope $C_{n}$ should be defined as a function of applied power $P_{i n, n}$ for calculation in the next second measurement.

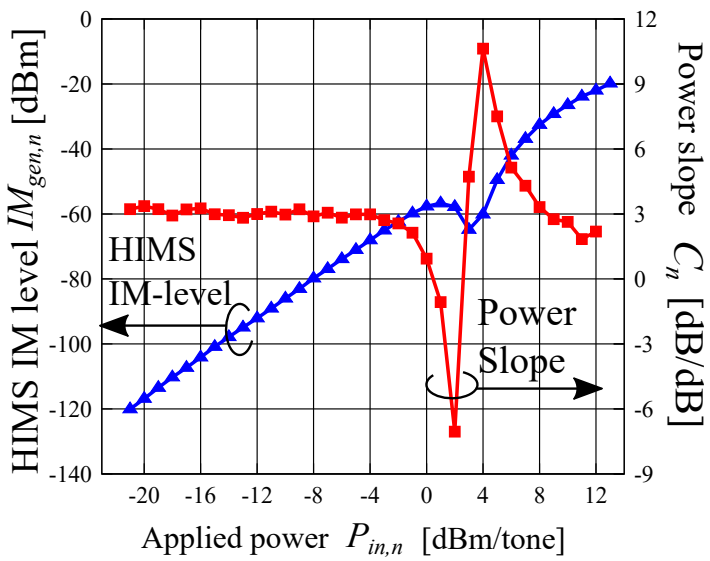

Fig. 7. IM characteristics $I M_{g e n, n}$ and power slope $C_{n}$ of HIMS as a function of applied power $P_{i n, n}$ in the first measurement.

\section{Second measurement}

In the second measurement, HIMS is installed on AUT; NCM is performed as shown in Fig. 2(b). In this case, the 
sensing antenna \#1 in Fig. 5 is connected to the PIM-tester; AUT \#2 is connected to a termination via HIMS. The observed IM level $I M_{r e}$ is expressed as the following equation on a decibel scale, where $P_{i n, j}$ is output from the PIM tester.

$$
\begin{aligned}
I M_{r e}= & I M_{g e n, j}-\int_{P_{i n, j}-\left|S_{c . f_{1}}\right|}^{P_{i n, j}} \frac{2}{3} C_{n}(P) d P \\
& -\int_{P_{i n, j}-\left|S_{c . f_{2}}\right|}^{P_{i n, j}} \frac{1}{3} C_{n}(P) d P+S_{c . f_{i m}}
\end{aligned}
$$

Therein, coupling losses between the sensing antenna and AUT at $f_{1}, f_{2}$, and $f_{i m}$ are, respectively, $S_{c . f_{1}}, S_{c . f_{2}}$, and $S_{c . f_{i m}}$. The first term on the right side $I M_{g e n, j}$ is an IM level caused by HIMS when $P_{i n, j}$ is applied directly to it. In other words, it is the result of the first measurement when the applied power is $P_{i n, j}$. The second and third terms represent the decrease of the generated IM because of the decrease of applied power at the respective transmitting frequencies of $f_{1}, f_{2}$. As described above, power slope $C_{n}$ is a function of the applied power. It is calculated by integration over a range to express the total decrease. The fourth term shows the coupling loss that is incurred when the generated IM is received by the sensing antenna. If the transmitted signals $f_{1}, f_{2}$ and the IM signal $f_{i m}$ are mutually close, then coupling losses can be approximated as $S_{c . f_{1}} \approx S_{c . f_{2}} \approx S_{c . f_{i m}}$. This approximation is natural because these test frequencies are in antenna bandwidth. Denoting these losses as $S_{c}$, equation (2) enables its solution as a transcendental equation for quantity $S_{c}$.

Table II presents the calculated power slope in the first measurement using HIMS. For these discrete samples, equation (2) is discretized as shown below.

$$
\begin{array}{r}
h\left(S_{c}\right)=I M_{g e n, j}-I M_{r e}-\sum_{n=i}^{j-1} C_{n} \Delta P_{n}+S_{c} \\
\Delta P_{n}=P_{i n, n+1}-P_{i n, n}
\end{array}
$$

In those equations, "i" is an arbitrary integer label; $S_{C}$ is expressed as $\sum_{n=i}^{j-1} \Delta P_{n}=S_{C}$. The solution of transcendental equation $h\left(S_{c}\right)=0$ is given as coupling loss $S_{c}$. As described above, IM signal observed in this case must be dominated by the one caused by HIMS. For that reason, a sufficiently highlevel standard is required as HIMS.

Table III presents comparison of an estimated coupling loss (Est.) based on equation (3) with a measured coupling loss (Meas.) using VNA when the distance between the sensing antenna and AUT is $d=160,210,260 \mathrm{~mm}$. In this case, output power from PIM tester is $P_{i n, j}=10 \mathrm{dBm} /$ tone, and $I M_{g e n, j}=-26.5 \mathrm{dBm}$ is obtained from the result of the first measurement. The observed IM level is $I M_{r e}=$ $-90.2,-95.9,-101.7 \mathrm{dBm}$, respectively, at $d=160,210,260$ $\mathrm{mm}$. The estimated value (Est.) can be regarded as a value obtained when the frequency characteristics are negligible, whereas the VNA measurements (Meas.) are values including the exact frequency dependency at each frequency. The table shows that the coupling losses are estimated with a difference of $2.3 \mathrm{~dB}$ against the VNA measurements. In the estimation, existence of HIMS affects the radiation pattern of AUT because the gain of AUT is low, which is regarded as a possible cause of the difference. However, detailed discussions are left as a subject for future work.

TABLE II

POWER SLOPE $C_{n}$ CALCULATED THROUGH THE FIRST MEASUREMENT

\begin{tabular}{c|c|c|c}
\hline Label & $P_{i n, n}$ & $I M_{g e n, n}$ & $C_{n}$ \\
\hline \hline 1 & $-21 \mathrm{dBm} /$ tone & $-120.1 \mathrm{dBm}$ & 3.2 \\
2 & $-20 \mathrm{dBm} /$ tone & $-116.8 \mathrm{dBm}$ & 3.4 \\
$\ldots$ & $\ldots$ & $\ldots$ & $\cdots$ \\
$\mathrm{i}$ & $P_{i n, i}$ & $I M_{g e n, i}$ & $C_{i}$ \\
$\ldots$ & $\ldots$ & $\ldots$ & $\cdots$ \\
$\mathrm{j}$ & $P_{i n, j}$ & $I M_{g e n, j}$ & $C_{j}$ \\
$\ldots$ & $\cdots$ & $\cdots$ & $\cdots$ \\
\hline
\end{tabular}

TABLE III

ESTIMATED COUPLING LOSS (EST.) BASED ON EQUATION (3) AND MEASURED COUPLING LOSS (MEAS.) USING VNA IN THE SECOND MEASUREMENT FOR THE BASIC SINGLE-ELEMENT MODEL SHOWN IN FIG. 5 .

\begin{tabular}{c|cccc}
\hline Distance $d$ & Est. & Meas. $\left(f_{1}\right)$ & Meas. $\left(f_{2}\right)$ & Meas. $\left(f_{\text {pim }}\right)$ \\
\hline \hline $160 \mathrm{~mm}$ & $-16.1 \mathrm{~dB}$ & $-14.6 \mathrm{~dB}$ & $-16.4 \mathrm{~dB}$ & $-14.4 \mathrm{~dB}$ \\
$210 \mathrm{~mm}$ & $-17.5 \mathrm{~dB}$ & $-16.3 \mathrm{~dB}$ & $-17.9 \mathrm{~dB}$ & $-15.2 \mathrm{~dB}$ \\
$260 \mathrm{~mm}$ & $-18.9 \mathrm{~dB}$ & $-17.5 \mathrm{~dB}$ & $-18.4 \mathrm{~dB}$ & $-17.5 \mathrm{~dB}$ \\
\hline
\end{tabular}

\section{Third measurement}

In the third measurement, HIMS is removed from AUT; NCM is performed for only AUT, as shown in Fig. 2(c). In this case, sensing antenna \#1 in Fig. 5 is connected to the PIM tester; AUT \#2 is terminated. The power applied to AUT $P_{A U T}$ in NCM is expressed as follows in the decibel scale.

$$
P_{A U T}=P_{i n}+S_{c}
$$

In that equation, $P_{i n}$ is the output power from the PIM tester. A generated PIM is also attenuated by the coupling loss. It is received by the sensing antenna. Therefore, the generated PIM level PIM Est.DM is expressed as follows in the decibel scale.

$$
P I M_{\text {Est.DM }}=P I M r e-S_{c}
$$

In that equation, $P I M_{r e}$ is a PIM level observed by the PIM tester. In this way, we enable estimation of the DM-value from NCM-value based on equations (4) and (5) using coupling loss $S_{c}$, which has already been estimated in the second measurement. This is the main process of compensation from the NCM-value to the DM-value. It is denoted as DM-NCM conversion in this paper.

Fig. 8 shows DM-NCM conversion results for the model shown in Fig. 5. PIM Est.DM is the estimated DM-value by NCM-value through the above process. It is calculated using coupling loss $S_{\text {c.Est. }}$ estimated by the second measurement. Because a sensing antenna has excellent low-PIM characteristics, residual noise is always below $-130 \mathrm{dBm}$ in this case. The directly measured DM-value is $P I M_{D M}$, which is found by connecting the PIM tester directly to AUT \#2 in Fig. 5. The estimated DM-value PIM Est.DM can be confirmed to agree 
with the measured DM-value $P I M_{D M}$, with maximum error of about $3 \mathrm{~dB}$. As a reference, the result estimated DM-value $P I M_{E s t . D M}^{\prime}$ calculated using the actual coupling loss $S_{C . m e a}$ measured by VNA at $d=160 \mathrm{~mm}$ is presented in Fig. 8, which is presented as the dotted line. Because the calculated result based on $S_{C . m e a}$ shows good agreement with the measured DM-value $P I M_{D M}$, we infer that the difference between estimated result $P I M_{E s t . D M}$ and measured result $P I M_{D M}$ is attributable to the small estimation error of the coupling loss obtained using HIMS in the second measurement.

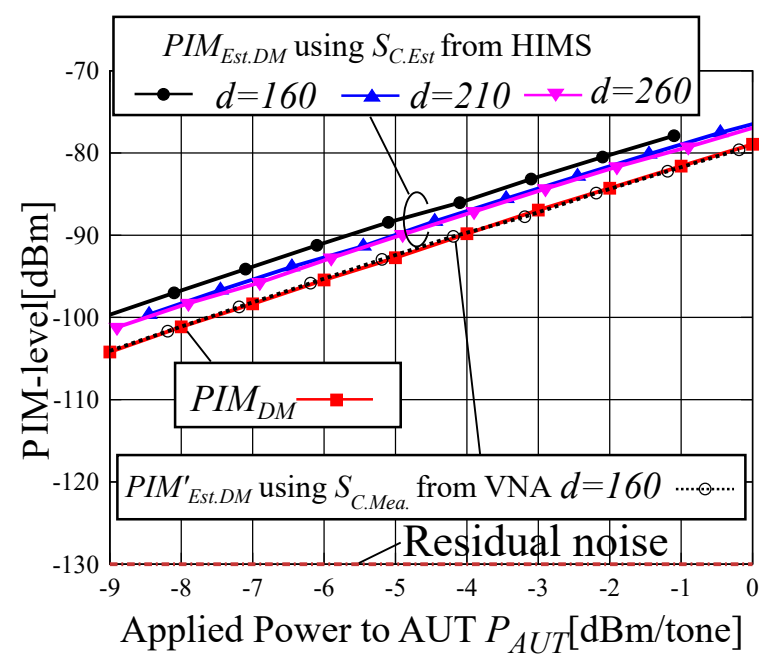

Fig. 8. Estimated DM-value PIM Est,DM from NCM using coupling loss $S_{C . E s t}$. estimated in the second measurement, and the estimated DMvalue PIM $M_{E s t, D M}^{\prime}$ using coupling loss $S_{C . M e a s}$. measured by VNA and measured DM-value $P I M_{D M}$ in the third measurement for the basic singleelement model shown in Fig. 5.

\section{Applicable Method for Array Antenna}

This section presents the practically applicable method for array antenna applied from the method proposed above. The method proposed in the preceding section is directly applicable to the far-field of array antenna. However, it is not practical from the viewpoint of requiring a large measurement space. Therefore, we consider feeding each antenna element independently with each excitation factor. As shown in Fig. 9, each antenna element is coupled with a small dipole probe in its vicinity. In this case, for the array fed with equal amplitude with phase difference $\delta$, each element is excited by each small dipole probe with a phase delay of $\delta$. In other words, probe \#P2, which excites antenna element \#E2, is fed with a phase delay of $\delta$ compared with probe \#P1. Also, $L_{a}, L_{s}, \delta$ represents the electrical length. To remove the effect of impedance mismatching caused by small dipole probes, a Wilkinson divider is used to feed with equal amplitude and with appropriate phase difference. In this symmetrical excitation method, DM-NCM conversion enables performance using the coupling loss similarly the basic single-element model shown in section 2. Detailed theoretical calculations are attached in Appendix A.

Fig. 10(a) shows model 2A, in which a PIM source is located in antenna element \#E2A, which is identical to that used in Fig. 5, whereas Fig. 10(b) shows model 2B, in which a PIM source shown in Fig. 3(b) is located at the origin of the feeding circuit. Both models are a two-element printed dipole array antenna fed by MSL-feeding-circuit with phasedifference $L_{\delta}$ adjusted to tilt the main beam to a 20 degree direction from the front. The Wilkinson divider comprises the MSL and a chip resistor. The feeding-circuit and Wilkinson divider are fabricated using the same dielectric substrate to HIMS, as shown in section 2 . In both models, small dipole probes with length of about $\lambda / 3$ are used. Also, an isolation cable (5B-002-18-07-113FBW; Candox System Inc.) is used to suppress the leakage current. Then, the whole circuit on the left side for detection of PIM for AUT is denoted as a sensing antenna.

Table IV presents results of the estimated coupling loss and the measured one using VNA in the second measurement. In this case, the output power from the PIM tester is $P_{i n, j}=10$ $\mathrm{dBm} /$ tone; the observed IM level is $I M_{r e}=-104.8,-104.0$ $\mathrm{dBm}$, respectively, for model $2 \mathrm{~A}$ and model $2 \mathrm{~B}$. The estimated coupling loss shows good agreement with the measured VNA.

Fig. 11 presents results obtained for DM-NCM conversion using the estimated coupling losses in the manner described in section 2. Unlike a basic single-element model, residual noise is caused mainly by sensing antenna, which has a complicated circuit in this case. For that reason, we must evaluate it carefully. It is recommended that residual noise be lower than the actual PIM level by $10 \mathrm{~dB}$ [12], Because this requirement is met in both models, the validity of the observed PIM level is confirmed. It is apparent that the estimated DM-value $P I M_{E s t . D M}$ are in good agreement with measured DM-value $P I M_{D M}$. In the result obtained for model $2 \mathrm{~B}$, little difference was found between the slope of PIM Est.DM and PIM $M_{D M}$, which is regarded as a possible cause of the fluctuation of coupling loss $S_{C}$, depending on the power range. However, detailed discussion of this matter is left as a subject for future work.

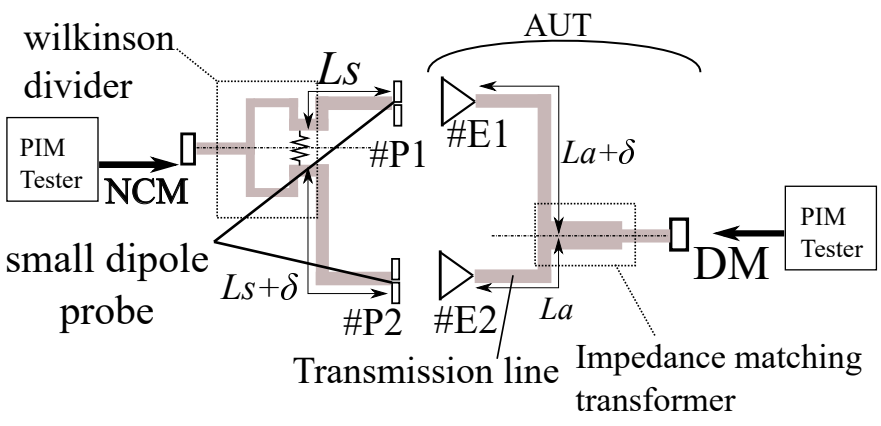

Fig. 9. Configuration for the array antenna measurement method using small dipole probes.

TABLE IV

COMPARISON OF AN ESTIMATED COUPLING LOSS (EST.) BASED ON EQUATION (3) WITH A MEASURED COUPLING LOSS (MEAS.) USING VNA IN THE SECOND MEASUREMENT FOR ARRAY ANTENNA MODELS

\begin{tabular}{c|cccc}
\hline Model & Est. & Meas. $\left(f_{1}\right)$ & Meas. $\left(f_{2}\right)$ & Meas. $\left(f_{\text {pim }}\right)$ \\
\hline \hline Model 2A & $-19.7 \mathrm{~dB}$ & $-19.0 \mathrm{~dB}$ & $-18.0 \mathrm{~dB}$ & $-21.3 \mathrm{~dB}$ \\
Model 2B & $-19.5 \mathrm{~dB}$ & $-19.5 \mathrm{~dB}$ & $-18.2 \mathrm{~dB}$ & $-21.6 \mathrm{~dB}$ \\
\hline
\end{tabular}




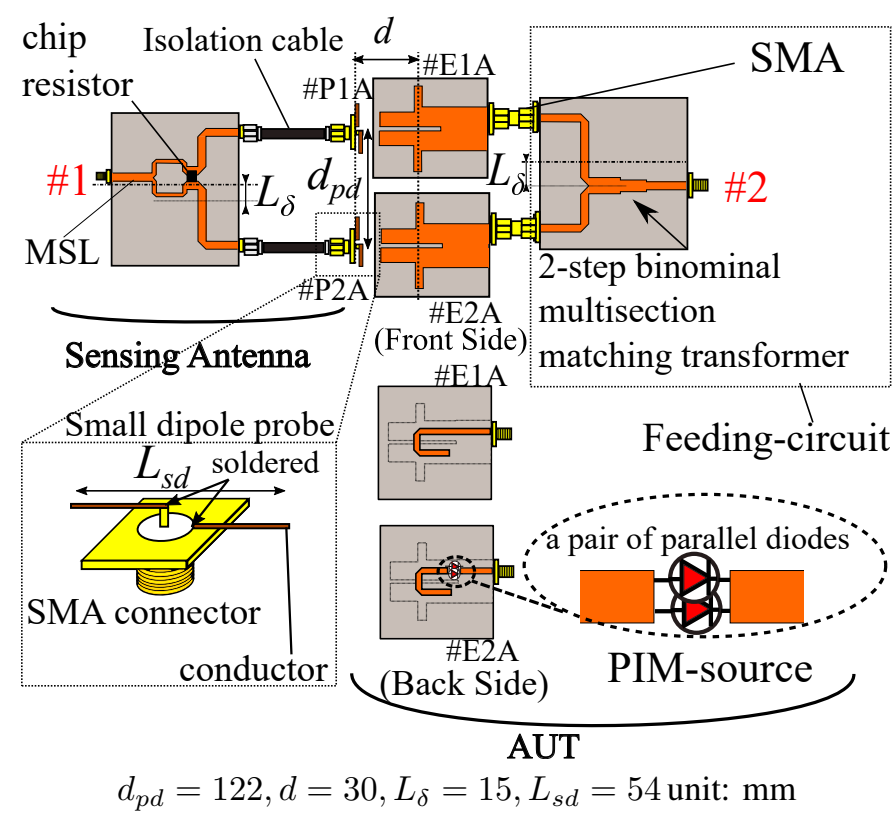

(a) Array antenna model with PIM source in antenna element (model 2A)

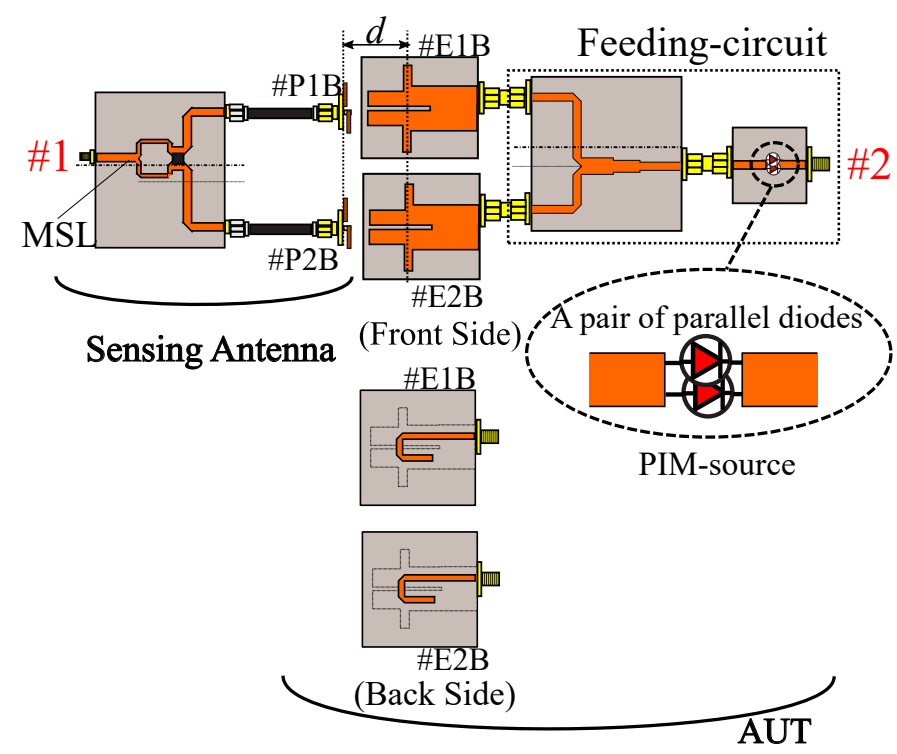

(b) Array antenna model with PIM source at the origin of the feeding circuit (model 2B)

Fig. 10. Configuration of array antenna models.

\section{CONCLUSION}

As described in this paper, we proposed a novel method to estimate coupling loss using a high-level IM standard (HIMS) for non-contact PIM measurement for antenna. As a result, the proposed measurement enables estimation of the practical PIM characteristics in a complete one-port non-contact measurement. Its validity is confirmed through PIM measurement of a printed dipole antenna using a HIMS consisting of a diode. In addition, this report presents description of an applicable measurement method for array antenna applied from the proposed method. Specifically, a small dipole probe was used for each element. It was excited with an appropriate phase difference depending on the excitation factor. Experimentally obtained

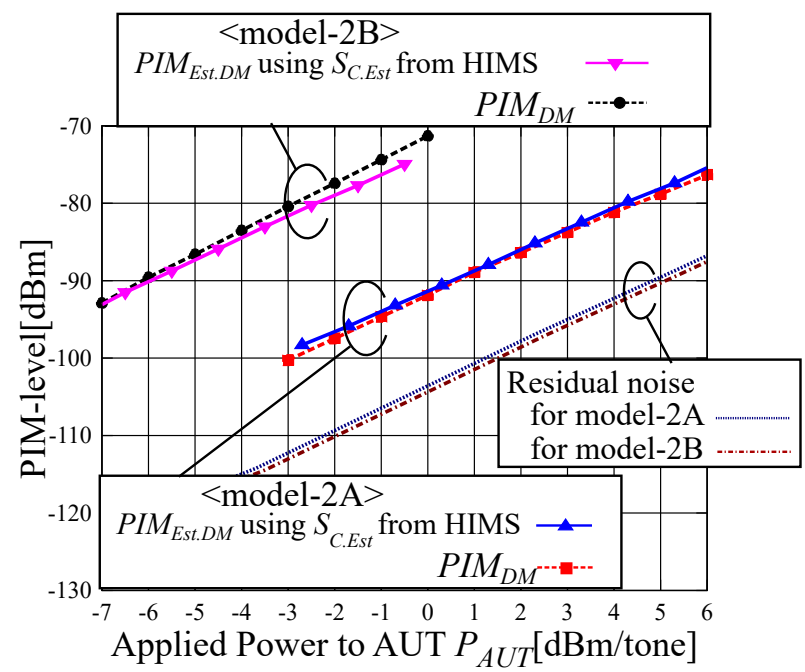

Fig. 11. Estimated DM-value PIM $M_{E s t, D M}$ from NCM using coupling loss $S_{C . E s t}$. estimated in the second measurement and measured DM-value $P I M_{D M}$ in the third measurement for array antenna models shown in Fig. 10

results show that the observed PIM level enabled similar conversion to practical PIM characteristics as well as for array antenna. Development of an advanced measurement system that is applicable for all array antennas with a complicated feeding-circuit is regarded as a subject to be undertaken for future work.

\section{APPENDIX A}

\section{THEORETICAL CALCULATION FOR AN ARRAY ANTENNA MEASUREMENT SYSTEM}

In this section, all parameters are calculated on a linear scale unless stated otherwise. Fig. 12 is a modified Fig. 9, where all the ends of the array circuit are labeled as \#m $(m=1 \ldots)$. A PIM source located at \#5 is denoted as PIM-Source-A. One located at \#6 is defined as PIM-Source-C. Transmitting powers are delivered from the right side in DM, whereas transmitting powers are delivered from the left side in NCM. Assuming that the applied voltage from the PIM tester is $V_{i n}$, then the voltage applied to each PIM source in DM and NCM is calculated as presented below.

$$
\begin{aligned}
V i_{D . A} & =\dot{V_{i n}} \dot{S_{56}} \\
V i_{N . A} & =\dot{V_{i n}}\left\{\dot{S_{31}} \dot{S_{53}}\left(1+\dot{S_{55}}\right)+\dot{S_{21}} \dot{S_{42}} \dot{S_{54}}\right\} \\
V i_{D . C} & =\dot{V_{i n}} \\
V i_{N . C} & =\dot{V_{i n}}\left\{\dot{S_{21}} \dot{S_{42}} \dot{S_{64}}+\dot{S_{31}} \dot{S_{53}} \dot{S_{65}}\right\}
\end{aligned}
$$

Therein, $V i_{D . A}$ and $V i_{N . A}$ respectively denote the voltage applied to PIM source A in DM and NCM. Similarly, $V i_{D . C} a n d V i_{N . C}$ respectively stand for the voltages applied to PIM source C. Because it is a passive circuit, $S_{n m}=S_{m n}$ is obtained. If each coupling condition between each antenna element and probe is kept as equal, then $\dot{S_{42}}=\dot{S_{53}}$ is obtained. Both are denoted as $S_{C}^{\prime}$ : In addition, from the phase difference of the deed line, $\dot{S_{21}} \dot{S_{42}} \dot{S_{64}}=\dot{S_{31}} \dot{S_{53}} \dot{S_{65}}$, $\dot{S_{21}} S_{42} \dot{S}_{54}=-\dot{S_{31}} \dot{S}_{53} \dot{S}_{55}$ is obtained. In addition, for an ideal divider, $\left|\dot{S_{21}}\right|=\left|\dot{S_{31}}\right|=\left|\dot{S_{46}}\right|=\left|\dot{S_{56}}\right|=1 / \sqrt{2}$ is obtained. Consequently, equation (10) is obtained. 


\section{REFERENCES}

$$
\left|V i_{D . A} / V i_{N . A}\right|=\left|V i_{D . C} / V i_{N . C}\right|=1 /\left|S_{c}^{\prime}\right|
$$

This result indicates that the difference in power applied by the NCM and DM at each PIM source enables compensation by coupling loss $S_{C}^{\prime}$.

The generated PIM signal is received as presented below as

$$
\begin{aligned}
V r_{D . A} & =k_{A} \dot{S_{65}} \\
V r_{N . A} & =k_{A}\left\{\dot{S_{13}} \cdot S_{35}\left(1+\dot{S_{55}}\right)+\dot{S_{12}} \dot{S_{24}} \dot{S_{45}}\right\} \\
V r_{D . C} & =k_{C} \\
V r_{N . C} & =k_{C}\left\{\dot{S_{12}} \dot{S_{24}} \dot{S_{46}}+\dot{S_{13}} \dot{S_{35}} \dot{S_{56}}\right\}
\end{aligned}
$$

where $V r_{D . A}$ stands for the voltage, which is the PIM signal caused by PIM source A and received by the PIM tester in DM; $V r_{N . A}$ is one in NCM. In addition, $V r_{D . C}$ and $V r_{N . C}$ respectively stand for those of the PIM source $\mathrm{C}$ and received by the tester in DM and NCM. Also, $k_{A}, k_{C}$ respectively represent the magnitude of the level of PIM source A and PIM source $\mathrm{C}$, which are constants determined by the nonlinearity of the PIM source. Because the generated PIM signal is known to be equally distributed in [12], PIM levels distributed through each route can also be expressed as $k_{A}$ and $k_{C}$. Consequently, equation (15) is obtained in a manner similar to that explained above.

$$
\left|V r_{D . A} / V r_{N . A}\right|=\left|V r_{D . C} / V r_{N . C}\right|=1 /\left|S_{c}^{\prime}\right|
$$

In fact, $\left|S_{16}\right|=S_{C}^{\prime}$, as might be readily apparent. It is estimated using the proposed method (in second measurement). Equations (10) and (15) are converted to the decibel scale as shown below.

$$
\begin{array}{r}
V i_{N . t . d B}=V i_{D . t . d B}+S_{c . d B}^{\prime} \\
V r_{D . t . d B}=V r_{N . t . d B}-S_{c . d B}^{\prime} \\
t=A \text { or } C
\end{array}
$$

The first equation indicates that applied power to the PIM source in NCM is compensated to the one in DM using coupling loss $S_{c}^{\prime}$, which is synonymous with equation (4). The second equation indicates that observed PIM level in NCM is compensated to that in DM using coupling loss $S_{c}^{\prime}$, which is synonymous with equation (5). Therefore, the DM-NCM conversion enables conduct in a manner similar to the basic single-element model.

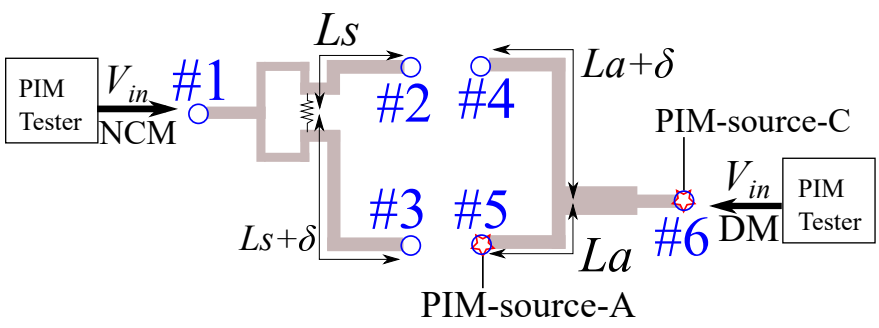

Fig. 12. Simplified diagram of Fig. 9 in which all terminals are labeled as $\# \mathrm{~m}(m=1 \cdots)$
[1] P. Bolli, S. Selleri, and G. Pelosi, "Passive intermodulation on large reflector antennas," IEEE Antennas Propag. Mag., vol.44, issue 5, pp.1320, 2002.

[2] Y. Patenaude, J. Dallaire, F. Menard, and S. Richard, "Antenna PIM measurements and associated test facilities," Proc. IEEE AP-S International Symposium (2001 Digest), Boston, MA, USA, pp.620-623, Jul. 2001.

[3] J. Sanford, "Passive intermodulation considerations in antenna design," Proc. IEEE AP-S International Symposium, Ann Arbor, MI, USA, pp.1651-1654, Jul. 1993.

[4] S.J. Chou, H.T. Chou, and L.R. Kuo, "Potential causes of PIM problems in the LTE outdoor base station multi-band antennas," Proc. 2016 Int Symp. Antennas Propag. (ISAP), Okinawa, Japan, pp.1080-1081, Oct. 2016.

[5] L. Chen, S. Lin, J. Qiu, X. Wang, and S. Fan, "Analysis and measurement of antenna passive intermodulation," 2010 International Conference on Electronics and Information Engineering, Kyoto, Japan, vol.2, pp.133135, Aug. 2010.

[6] P. Ansuinelli, A.G. Schuchinsky, F. Frezza, and M.B. Steer, "Passive intermodulation due to conductor surface roughness," IEEE Trans. Microw. Theory Tech., vol.66, issue 2, pp.688-699, Feb. 2018.

[7] C. Vicente, and H.L. Hartnagel, "Passive-intermodulation analysis between rough rectangular waveguide flanges," IEEE Trans. Microw. Theory Tech., vol.53, issue 8, pp.2515-2525, Aug. 2005.

[8] J. Henrie, A. Christianson, and W.J. Chappell, "Engineered passive nonlinearities for broadband passive intermodulation distortion mitigation," IEEE Microw. Wirel. Compon. Lett., vol.19, issue 10, pp.614-616, Oct. 2009.

[9] A.P. Shitvov, T. Olsson, B.E. Banna, D.E. Zelenchuk, and A.G. Schuchinsky, "Effects of geometrical discontinuities on distributed passive intermodulation in printed lines," IEEE Trans. Microw. Theory Tech., vol.58, issue 2, pp.356-362, Feb. 2010.

[10] C. Vicente, D. Wolk, H.L. Hartnagel, B. Gimeno, V.E. Boria, and D. Raboso, "Experimental analysis of passive intermodulation at waveguide flange bolted connections," IEEE Trans. Microw. Theory Tech., vol.55, issue 5, pp.1018-1028, May 2007.

[11] J. Henrie, A. Christianson, and W.J. Chappell, "Prediction of passive intermodulation from coaxial connectors in microwave networks," IEEE Trans. Microw. Theory Tech., vol.56, issue 1, Jan. 2008.

[12] RF connectors, connector cable assemblies and cables intermodulation level measurement, IEC standard 620371-1, Jul. 2012.

[13] J.R. Wilkerson, I.M. Kilgore, K.G. Gard, and M.B. Steer, "Passive intermodulation distortion in antennas," IEEE Trans. Antennas Propag., vol.63, issue 2, pp.474-482, Feb. 2015.

[14] K. Irie, N. Kuga, and K. Cho, "PIM-linearity improvement by the size of a diode mounting hole," IEICE Electron. Expr., vol.8, no.14, pp.11981203, Jul. 2011.

[15] M. Wang, I.M. Kilgore, M.B. Steer, and J.J. Adams, "Characterization of intermodulation distortion in reconfigurable liquid metal antennas," IEEE Antennas Wirel. Propag. Lett., vol.17, issue 2, pp.279-282, Feb. 2018.

[16] J.B. Yan, S. Yong, and J.T. Bernhard, "Intermodulation and harmonic distortion in frequency reconfigurable slot antenna pairs," IEEE Trans. Antennas Propag., vol.62, issue 3, pp.1138- 1146, Mar. 2014.

[17] S. Hienonen, V. Golikov, P. Vainikainen, and A.V. Raisanen, "Nearfield scanner for the detection of passive intermodulation sources in base station antennas," IEEE Trans. Electromag. Compat. vol.46, issue 4, pp.661-667, Nov. 2004.

[18] A.P. Shitvov, D.E. Zelenchuk, and A.G. Schuchinsky, and V.F. Fusco, "Passive intermodulation generation on printed lines: near-field probing and observation," IEEE Trans. Microw. Theory Tech., vol.56, issue 12, pp.3121-3128, Dec. 2008.

[19] D. Ishibashi, and N. Kuga, "Non-contact PIM evaluation method using a standing wave coaxial tube," IEICE Trans. Electron., vol.E95-C, no.9, pp.1508-1514, Sep. 2012.

[20] H. Suzuki, and N. Kuga, "Non-contact PIM measurement of large samples using a small anechoic box," Proc. 2016 URSI Asia-Pacific Radio Science Conference (URSI AP-RASC), Seoul, Korea (South), pp.1332-1333, Aug. 2016.

[21] R. Okawa, and N. Kuga, "Reverse-PIM extraction in non-contact antenna-PIM measurement," Proc. 2017 IEEE Asia Pacific Microwave Conference (APMC), Kuala Lumpur, Malaysia, pp.1163-1167, Nov. 2017.

[22] T. Kimino, and N. Kuga, "Basic consideration on non-contact localization for a PIM source in array antenna” 2020 Int. Symp. Antennas Propag. (ISAP), (Online), pp.395-396, Jan. 2021. 
[23] K. Takada, N. Kuga, and K. Cho, "An experimental consideration on the effect of antenna gain on the observed PIMs for an external PIM-source," 2012 Int. Symp. Antennas Propag. (ISAP), Nagoya, Japan, pp.991-994, Oct. 2012. 